Archives of Urology

ISSN: 2638-5228

Volume 1, Issue 2, 2018, PP: 44-47

\title{
Enteric Non Urachal Adenocarcinoma of the Bladder: A Case Report \\ Carolina D’Elia ${ }^{1 *}$, Emanuela Trenti ${ }^{1}$, Christine Mian² ${ }^{2}$, Esther Hanspeter ${ }^{2}$, Dorjan Huqi ${ }^{1}$ Armin Pycha ${ }^{1,3}$ \\ ${ }^{1}$ Urology Department, Bolzano General Hospital, Bolzano, Italy. \\ ${ }^{2}$ Pathology Department, Bolzano General Hospital, Bolzano, Italy. \\ ${ }^{3}$ Sigmund Freud Private University, Vienna, Austria. karolinedelia@gmail.com \\ *Corresponding Author: Carolina D'Elia, MD, FEBU, PhD, Urology Department, Bolzano Central Hospital, Bolzano, Italy.
}

\begin{abstract}
Introduction: Adenocarcinoma of the bladder is a rare bladder cancer subtype, accounting for about 0.5-2\% of all subtypes of cancer.

We report the case of a 80 year old patient with an enteric adenocarcinoma of the bladder, grown on a tubulovillous adenoma.

Case Report: A 80 year old man, with a Charlson comorbidity index 2, underwent a computer tomography (CT) angiography of the abdomen and legs due to the presence of occlusive vascular disease, that showed the presence of a focal contrast enhancement of $12 \mathrm{~mm}$ at the bladder dome. The patient underwent a transurethral resection of a bladder tumour and the histological examination revealed the presence of an enteric adenocarcinoma of the bladder (low grade) growth on a tubulovillous adenoma (pT1 G2) (Figure 1-4).
\end{abstract}

The patient was treated conservatively and after 34 months of follow up showed no evidence of disease and was asymptomatic.

Keywords: bladder cancer, enteric, villous adenoma, adenocarcinoma

\section{INTRODUCTION}

Bladder cancer is the ninth more common cancer, with estimated 430.000 new cases in 2012 and 165.000 deaths worldwide [1].

Ninety percent of bladder cancers are urothelial carcinomas [2], whereas adenocarcinoma of the bladder is a rare subtype, accounting for about $0.5-2 \%$ of all subtypes of cancer [3]. As a risk factor for the development of an adenocarcinoma the clam-cystoplasty is well known, adenocarcinomas develope frequently at the enterovesical anastomosis [5]. The leading symptom in this subtype is macroscopic haematuria, followed by storage symptoms and mucosuria as shown in a series of 72 patients affected by primary adenocarcinoma seen at the MD Anderson Cancer Center [4].

Management of these tumors represents a challenge for the urologist, due to the possible differential diagnosis with urachus adenocarcinoma,intestinal infiltration and due to the lack of response to standard adjuvant therapies [6].

We report the case of a 80 years old patient with an enteric adenocarcinoma of the bladder grown on a tubulovillous adenoma.

\section{CASE REPORT}

A 80 year old man, ex smoker, with an ECOG performance status 1 and Charlson comorbidity index 2, BMI 26, has come to our attention due to the incidental discovery of bladder neoplasm.

The patient suffered from chronic pulmonary obstructive disease and persistent atrial fibrillation (NYHA 1; ASA score 2); therefore he was treated with Warfarin. 


\section{Enteric Non Urachal Adenocarcinoma of the Bladder: A Case Report}

The patient underwent a CT angiography of the abdomen and legs due to the presence of occlusive vascular disease; the examination showed the presence of a focal contrast enhancement of $12 \mathrm{~mm}$ at the bladder dome and arterial stenosis of the posterior tibial artery on the right leg.

The patient did not report any history of pain, hematuria or other urological symptoms; at the physical examination the abdomen was not distended and no pain was reported. At the digitorectal exploration the prostate was not suspicious. Urinary cytology was positive for a high grade lesion.

A flexible cystoscopy showed the presence of a urethral stenosis and a pedunculated lesion located on the posterior bladder wall.

Preoperative chest radiography and blood tests were regular; creatinine level was $0.83 \mathrm{mg} / \mathrm{dl}$.
The patient underwent, subsequently, a transurethral bladder resection (TUR-B) of the $1.2 \mathrm{~cm}$ exophytic tumour of the posterior bladder wall.

The post- operative course was uneventful and the patient was discharged on the first post-operative day.

The histological examination revealed the presence of an enteric adenocarcinoma of the bladder (low grade) growth on a tubulovillous adenoma (pT1 G2) (Figure 1-4).

The adenomatous portion of the lesion presented with elongated villi lined by intestinal type epithelia (Fig.1,2), whereas the infiltrating enteric adenocarcinoma showed intestinal type glands with pseudostratified columnar cells and nuclear atypia, closely resembling colorectal adenocarcinoma, and necrotic foci (Fig3-4).

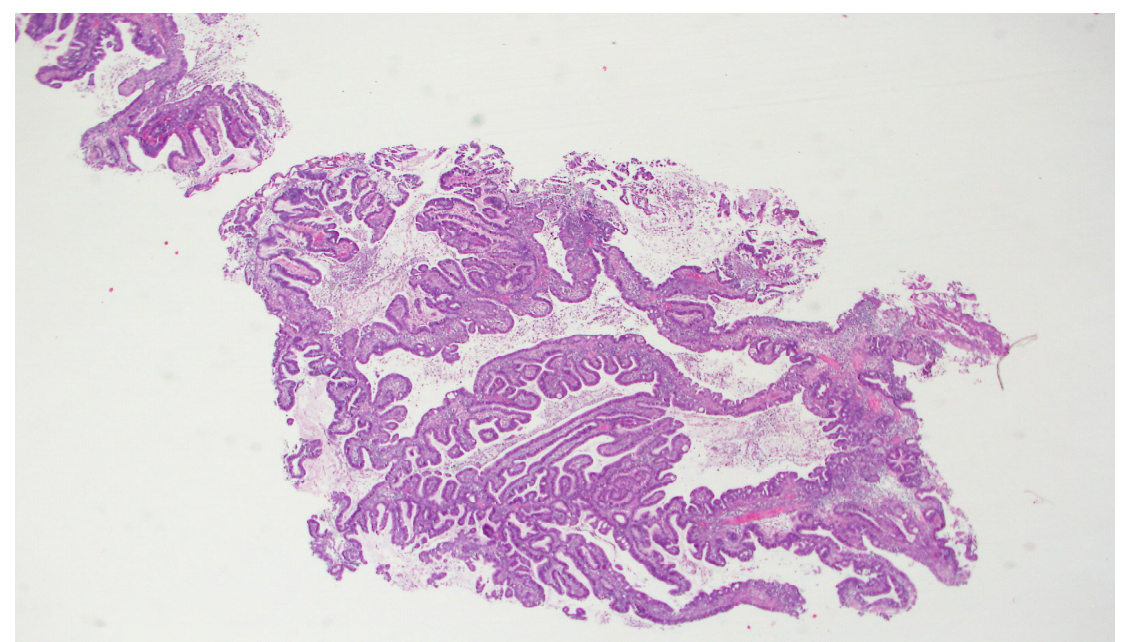

Fig 1. Overview of the adenomatous portion of the lesion with elongated villi lined by intestinal-type epithelium

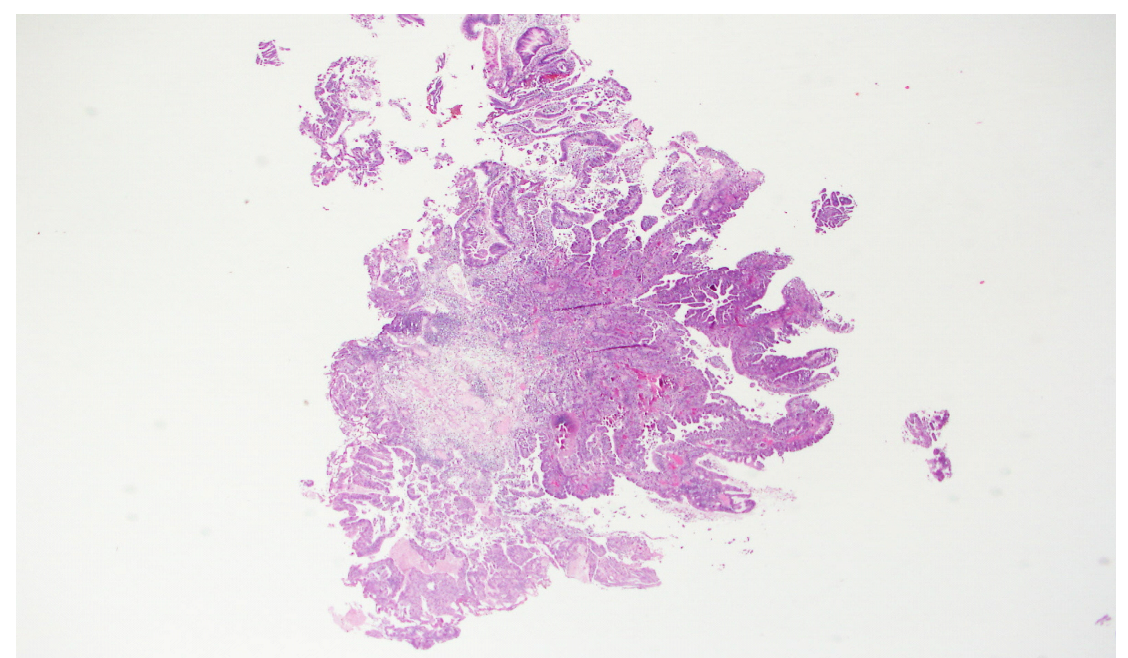

Fig 2. Overview of the adenoma with high grade dysplasia and adenocarcinoma infiltrating the lamina propria 


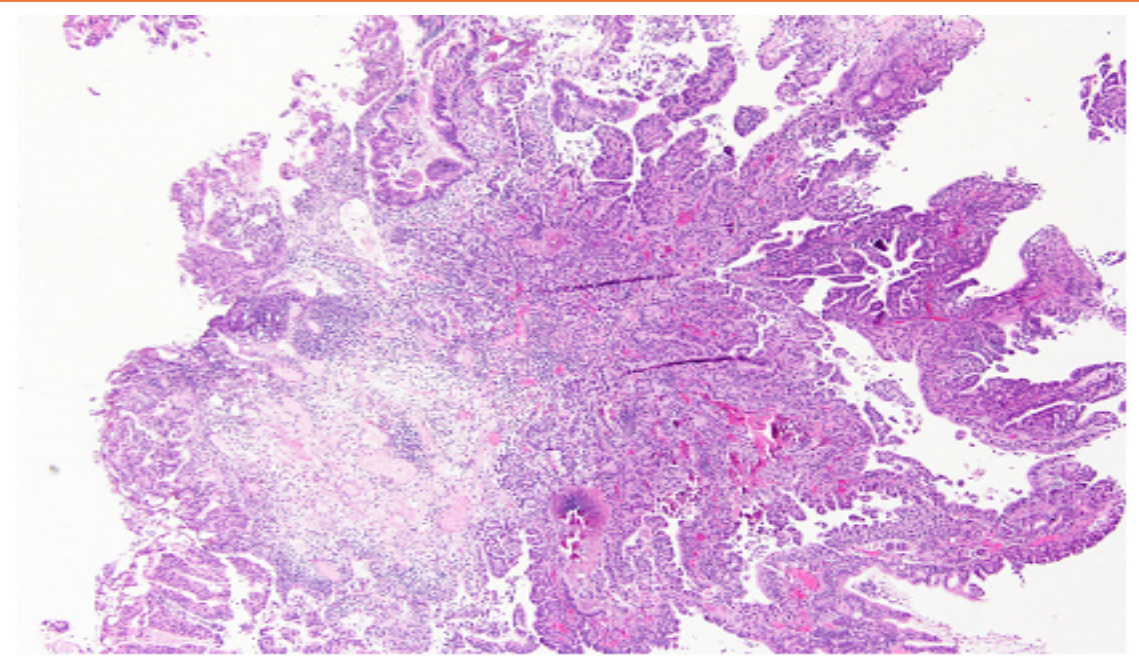

Fig 3. Portion of infiltrating adenocarcinoma of the enteric type with intestinal-type glands

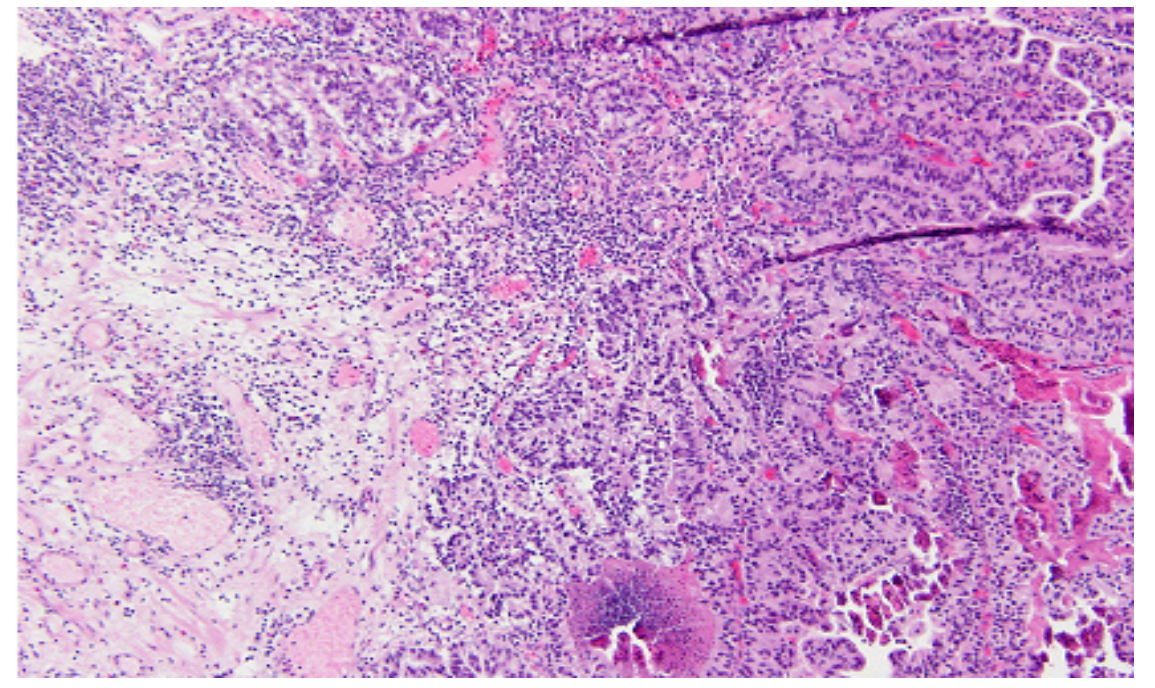

Fig 4. Portion of infiltrating adenocarcinoma of the enteric type; in the lower part of the picture a focus of necrosis.

In the second look TUR-B the histological evaluation was negative. After discussion of the case, the patient was followed with a cystoscopy and bladder random biopsies at 3 and 6 months, which resulted negative.

After 34 months of follow up the patient showed no evidence of disease and was asymptomatic.

\section{DiscusSiON}

Adenocarcinoma of the bladder is a rare subtype of bladder cancer, accounting for less than $2 \%$ of all bladder tumors [7]; literature data about its treatment and prognosis are sparse and not conclusive.

The most frequent presentation symptom of this disease is hematuria [4], as reported in the series of Grignon et al, who found a $87.5 \%$ rate of hematuria as main symptom in the non-urachal tumours; nevertheless, in our case the tumour was detected incidentally, during a CT scan performed for presence of occlusive vascular disease.

In our case, a low grade adenocarcinoma developed on a tubulovillous adenoma of the bladder.

Villous adenomas of the bladder are rare bladder neoplasm arisen generally from cloacal rests located in the bladder [8] or by a malignant transformation due to a chronic irritation, causing glandular metaplasia, up to dysplasia and subsequent carcinoma [9].

Villous adenoma of the bladder show generally a good prognosis, even when associated with adenocarcinoma, as shown by a series of 23 patients ( 15 patients with isolated villous adenoma and 8 patients with coexistent adenocarcinoma) with a long term follow up of 9.9 years; only one patient, with associated adenocarcinoma, developed lung metastases [10]. 
Montironi et al [10] suggest an aggressive treatment, in patients with coexisting adenocarcinoma, because these patients are at risk of developing recurrence or distant metastases.

A recent review, Porten et al. recommended early cystectomy in patients with adenocarcinoma and other rare variants, like pure squamous, sarcomatoid, plasmacytoid or micropapillary disease [11].

In our case, the low grade adenocarcinoma was discovered incidentally and the patient was treated with TUR-B and a second look TUR-B; after 34 months of follow up the patient did not show evidence of disease recurrence and was asymptomatic.

Nevertheless, the treatment of these rare histologic variants of bladder carcinoma represents a challenge for the urologist and treatment and follow up should be tailored on the characteristics of the patient himself.

\section{REFERENCES}

[1] Antoni S, Ferlay J, Soerjomataram I, et al: Bladder Cancer Incidence and Mortality: A Global Overview and Recent Trends. Eur Urol. 2017; 71(1):96-108.

[2] Blaveri E, Simko JP, Korkola JE, et al: Bladder cancer outcome and subtype classification by gene expression.Clin Cancer Res. 2005;11(11):404455.

[3] Dadhania V, Czerniak B, Guo CC: Adenocarcinoma of the urinary bladder Am J Clin Exp Urol 2015; 3(2):51-63.
[4] Grignon DJ, Ro JY, Ayala AG, et al: Primary adenocarcinoma of the urinary bladder. A clinicopathologic analysis of 72 cases. Cancer 1991; 67(8):2165-2172.

[5] Castellan M, Gosalbez R, Perez-Brayfield $M$, et al: Tumor in bladder reservoir after gastrocystoplasty. J Urol 2007;178(4 Pt 2):17711774.

[6] Bertz S, Hartmann A, Knüchel-Clarke R et al: Specific types of bladder cancer. Pathologe 2016; 37(1): 40-51.

[7] Kantor AF, Hartge P, Hoover RN, Fraumeni JF Jt Epidemiological characteristics of squamous cell carcinoma and adenocarcinoma of the bladder. Cancer Res. 1988; 48(13): 3853-3855.

[8] Wang J, Manucha V. Villous Adenoma of the Urinary Bladder: A Brief Review of the Literature. Arch Pathol Lab Med. 2016;140(1): 91-93.

[9] Adegboyega PA, Adesokan A. Tubulovillous adenoma of the urinary bladder. Mod Pathol. 1999; 12(7):735-738.

[10] Cheng L, Montironi R, Bostwick DG Villous adenoma of the urinary tract: a report of 23 cases, including 8 with coexistent adenocarcinoma. Am J Surg Pathol. 1999 Jul;23(7):764-771.

[11] Porten SP, Willis D, Kamat AM: Variant histology: role in management and prognosis of nonmuscle invasive bladder cancer. Curr Opin Urol. 2014; 24(5): 517-523.

Citation: Carolina D'Elia, Emanuela Trenti, et al. Enteric Non Urachal Adenocarcinoma of the Bladder: $A$ Case Report. Archives of Urology. 2018; 1(2): 44-47.

Copyright: (C) 2018 Carolina D’Elia, Emanuela Trenti, et al. This is an open access article distributed under the Creative Commons Attribution License, which permits unrestricted use, distribution, and reproduction in any medium, provided the original work is properly cited. 